\title{
Control de un péndulo de Furuta, una revisión del estado del arte.
}

\author{
Control and friction of Furuta pendulum, state of art. \\ Giovanni Triana García, José Tomas Buitrago, José Miguel Ramírez \\ Escuela de Ingeniería Eléctrica y Electrónica, Grupo de Investigación en control e instrumentación \\ industrial GICI, Universidad del Valle, Santiago de Cali, Colombia.
}

\begin{abstract}
Resumen - En este trabajo se presenta un estado del arte sobre las estrategias de modelado y control utilizadas en un péndulo invertido rotacional. Se realiza una breve descripción de cada una de estas estrategias desde la creación del péndulo de Furuta hasta la actualidad, con especial atención a la consideración o no de la fricción en la concepción del control. Se revisan los principales aportes a nivel mundial y en Colombia.
\end{abstract}

Palabras claves - Péndulo Invertido, Estabilización, Estrategias de control, Mecánica clásica.

Abstract - This work shows a state of art about the different modeling and control strategies for the inverted rotational Pendulum. A brief description of each of them from the creation of the Furuta Pendulum to the present day was made, we pay great interest of Friction phenomena in the control strategies, highlighting the whole world, include Colombia.

Key Word - Classic mechanics, Control strategies, Inverted Pendulum, Stabilization.

\section{INTRODUCCIÓN.}

El péndulo es un objeto físico consistente en una masa unida mediante una delgada cuerda o barra a un pivote, el sistema es libre de moverse realizando un balanceo alrededor de su punto de equilibrio estable o natural, en condiciones naturales y sin influencia de fuerzas el dispositivo estará en dicha posición, este objeto tan conocido desde la antigüedad ha ido evolucionando y actualmente se utiliza en los laboratorios de control como punto de prueba o de referencia para el análisis y estudio de estrategias de control lineal y no lineal. Uno de los péndulos más conocidos y estudiados es el péndulo invertido planar [1], el cual consiste en un carro que lleva un péndulo en su parte superior y se desplaza sobre un riel (ver figura 1).



Figura 1. Péndulo invertido simple planar [1].
Aquí se presentan limitaciones en el movimiento del carro y por lo tanto hay restricciones; este problema fue sorteado en el año 1991 con el reemplazo del carro sobre riel por un motor eléctrico que maneja directamente un brazo horizontal [2], como se muestra en la figura 2.

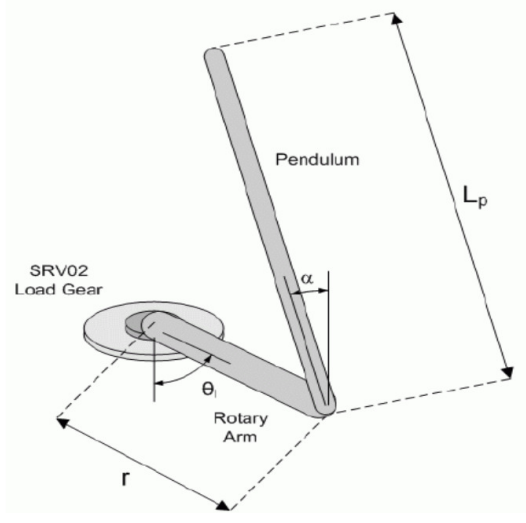

Figura 2. Péndulo invertido rotacional simple o de Furuta. (Quanser Consulting, 102 George Street, Hamilton, Ontario, Canada).

Este dispositivo es conocido como el péndulo invertido rotacional simple o de Furuta y su conformación se da por dos elementos, el primero de ellos se mueve únicamente en un plano horizontal y es controlado por un motor eléctrico de corriente continua CC (sistema actuado), el segundo elemento corresponde al péndulo o brazo colgante que es libre de moverse en un plano vertical (sistema sub-actuado). Por lo tanto, se trata de un sistema con dos grados de libertad y una entrada de control; el péndulo invertido rotacional simple se ha convertido en un punto de referencia para el análisis y estudio de estrategias de control clásico y moderno, lineal y no lineal, al igual que es utilizado con propósitos didácticos como el caso de la enseñanza de la teoría de control y el modelado de sistemas físicos [3]. En cuanto a la forma de obtener el modelado o el método utilizado, se puede lograr a partir de la mecánica clásica, ecuaciones de Euler-LaGrange, Newton iterado o grafos de Bond, entre otros. Su dinámica es altamente no lineal y erguido presenta inestabilidad en lazo abierto.

También existen péndulos dobles, los cuales han sido utilizados para demostrar las bondades de las ecuaciones en 
espacio de estados (K. Furuta y H. Kajiwara, 1980) y péndulos triples (K. Furuta, T. Ochiai, y N. Ono, 1984) donde la estabilización presenta un grado de dificultad alto por las incertidumbres en el modelado matemático.

Por otro lado, el interés generado en los sistemas subactuados ha conducido a un amplio estudio de los mismos con el propósito de reducir los costos en sistemas controlados automáticamente o sistemas robóticos; en ellos se desea disminuir el número de actuadores con el propósito de racionar potencia, señales, sensores, peso, etc. Esto se refleja en una mayor economía. También es muy utilizado en estudios de casos donde uno de los actuadores falla y el sistema se convierte en un sistema sub-actuado.

Para el control de un péndulo invertido rotacional simple se consideran generalmente dos etapas: la primera corresponde al levantamiento del péndulo desde su estado de equilibrio estable o natural, a este movimiento se le conoce como swing up, donde la mayoría de las veces se utiliza un análisis energético para obtener una estrategia de control que alcance este objetivo y luego se cambia o conmuta a la segunda etapa del problema que es buscar otra estrategia de control que se encargue del sostenimiento o estabilización del péndulo y debido al cambio de estrategia de control a ésta se le denomina control conmutado. Existen otras estrategias de control donde se logra el levantamiento y estabilización del péndulo con una misma estrategia, por lo que se le conoce como control sin conmutación. Estas estrategias de control por lo general se realizan sin considerar los efectos de la fricción.

\section{CONTROL DE PÉNDULOS INVERTIDOS}

En esta sección se expondrá en forma breve algunas de las estrategias de control encontradas en artículos reportados por la comunidad a nivel mundial y en Colombia, se han agrupado los artículos según características comunes tanto en el diseño de la estrategia de control como el de su objetivo, pero también se presentan tratando de respetar el orden cronológico en el momento en que fueron reportadas.

\section{A. Control basado en energías.}

En la actualidad, los diseñadores de estrategias de control utilizan la técnica basada en energía para el estudio de estabilidad de sistemas no lineales y como estrategia de control, ya que las funciones matemáticas que modelan los fenómenos naturales correspondientes a energías (cinética de translación, rotación, potencial gravitacional, etc.) son fáciles de describir, como también, se facilita el poder conocer los términos de un sistema que disipan energía, o aquellos que inyectan energía o si por el contrario la energía permanece constante, todo esto es importante para el control de dicho sistema.

Se procederá a realizar una descripción en forma breve algunos trabajos reportados, donde se utiliza una estrategia de control basado en concepciones energéticas.
En el año de 1996, Åström y Furuta [4] desarrollan un control para el levantamiento de un péndulo invertido rotacional simple basado en energías; la concepción del control es a partir del conocimiento de la energía necesaria para llevar el péndulo desde su posición de equilibrio natural (abajo), hasta su posición erguido en la parte superior, el comportamiento de la ley de control depende de la relación entre la aceleración máxima del pivote y la aceleración de la gravedad. En el año 2002 aparece una propuesta de una estrategia de control para sistemas electromecánicos sub actuados (entre ellos un péndulo invertido), en este trabajo se considera un control basado en la estructura Hamiltoniana (tesis doctoral Fabio GómezStern Aguilar, Universidad de Sevilla, 2002) [5]. La idea que se describe en este trabajo es el de un método de diseño de control basado en pasividad con asignación de interconexión y amortiguamiento, tal método es mejor conocido por sus siglas en inglés como IDA-PBC (Interconnection and Damping Assignment Passivity Based control); por medio de éste se logra el levantamiento y la estabilización del péndulo invertido, sin conmutación de la ley de control.

El análisis basado en energías no se limita al levantamiento del péndulo, también se aplica al diseño de control para lograr la estabilización; Sujit Nair y Naomi Ehrich Leonard, 2002 [6], ellos realizan el modelado con base en una aproximación del péndulo planar al péndulo de Furuta; utilizan el método de Lagrangiano controlado (equivalente al IDA-PBC) vía el moldeo de energía (energy Shaping) para la estabilización. Se prueba la estabilidad exponencial local del origen utilizando la energía controlada del sistema linealizado como una función de Lyapunov.

En al año 2007, se presta nuevamente atención al diseño de control basado en consideraciones energéticas; es así como Konstantin Machleidt, Jens Kroneis, y Steven Liu [7] logran la estabilización del péndulo de Furuta con el método de control no lineal basado en el Lagrangiano controlado. Por primera vez se tienen en cuenta los efectos de la fricción; se realiza simulación y experimentación. En este trabajo nuevamente se hace el estudio del modelo de un péndulo invertido planar y luego se aproxima a un péndulo invertido rotacional, se logra la estabilización del sistema con una entrada de control bastante pequeña comparada con la de un controlador lineal convencional.

Para este mismo año L. Freidovich, A. Shiriaev, F. Gordillo, F. Gómez-Estern y J. Aracil, 2007 [8], abordan el problema de las oscilaciones a alta frecuencia de un péndulo de Furuta alrededor del punto de equilibrio inestable en lazo abierto. Lo primero que se plantea es el diseño de un control basado en energías (energy Shaping) para la articulación sub actuada, luego se crea un control basado en la disipación de energía para lograr las oscilaciones en el elemento actuado, a esto se suma una acción de realimentación lineal auxiliar para la compensación de la energía; en este trabajo se implementa simulación y experimentación, encontrándose que existe una discrepancia en el valor angular de la articulación actuada y los autores realizan la anotación de que se 
produce porque no hay una compensación perfecta del torque de fricción.

En el 2008, K.J. Åström, J. Aracil y F. Gordillo [9] presentan un ingenioso método de una familia de controladores suaves para el levantamiento del péndulo de Furuta basado en energías (formación del Hamiltoniano) y se logra la estabilización sin control conmutado. Se encuentra una discrepancia, ya que en la experimentación se logra el levantamiento en 5 ciclos más de lo que muestra la simulación, esto se debe a que no se considera la fricción en el péndulo, así lo expresan los investigadores en el documento.

En el año 2009 Viroch Sukontanakarn y Manukid Parnichku [10] presentan un estudio donde se propone un control PD basado en energía para lograr el levantamiento, la estabilización se logra con un control LQR (Linear Quadratic regulator) en tiempo real; se realiza experimentación y simulación.

\section{B. Control por realimentación de estados.}

En este apartado se trata la descripción de las estrategias de control que se basan en la realimentación de estados. Esta estrategia se fundamenta en la escogencia de las ganancias de realimentación, ya que se relacionan directamente con el comportamiento del sistema bajo la acción de control.

En 1991 se propone un nuevo dispositivo llamado el péndulo invertido rotacional o de Furuta, Furuta et al [2], en donde se diseña una estrategia para el levantamiento o swing up, por medio de un control On-Off por realimentación de estados con información del plano de fase (conocido como control Bang-Bang). La estabilización se logra con un control LQ (Linear Quadratic); resulta ser un control robusto, incluso se implementó para un péndulo doble, pero los resultados no fueron reportados.

En el año 2002 S. Awtar, N. King, T. Allen and K. Craig [11], diseñan un péndulo invertido rotacional simple o de Furuta y la estabilización del mismo se logra utilizando una estrategia de control por realimentación de estados. Para el caso del swing up o levantamiento se utiliza un control basado en energías.

Yung-Chih Fu y Jung-Shan Lin [12], en el año de 2005 desarrollan un trabajo donde se explica el diseño no lineal Backstepping, cuyo objetivo es llevar el péndulo de Furuta desde su posición en equilibrio estable (posición abajo) hasta la posición de equilibrio inestable (posición arriba). Aquí se incluye un diseño basado en Lyapunov y se reduce a modelos de primer orden.

Para el 2006 Carlos Aguilar-Ibáñez, Octavio Gutiérrez F. y Humberto Sossa-Azuela [13], presentan un trabajo donde se realiza una conveniente realimentación parcial de estados, luego buscan la función candidata de Lyapunov para lograr la estabilización; se demuestra que este sistema es localmente asintóticamente estable alrededor del punto de equilibrio inestable, no se realiza simulación.
En el año 2014 se reporta un artículo presentado por Mandic P, Lazarevic M, et al [14]. Aquí primero se caracteriza la parte electromagnética y se obtiene el modelo, luego se estudia el péndulo invertido obteniendo también el modelo matemático y se considera el levantamiento del péndulo y la estabilización mediante una realimentación parcial de estados linealizado y un PID. Se realiza simulación y experimentación.

\section{Control por estructura variable.}

Este tipo de control no lineal que presenta conmutación a muy rápida velocidad, es muy utilizado por presentar robustez, pero en algunas ocasiones acarrea algunos tipos de problemas como el chattering. A continuación se describirá en forma breve algunos trabajos presentados en la comunidad de diseñadores de estrategias de control.

Jeong Ju Choi y Jong Shik Kim en el año 2003 [15] presentan el diseño de un control por modos deslizantes con realimentación de la salida y un control en la estabilización del péndulo por este medio, éste muestra un buen desempeño en el rechazo de disturbios e incertidumbres en el modelado; también se diseña una estrategia de control con observador de disturbios con modelado de error, por último se realiza una comparación con la estabilización por medio de un control LQ, demostrando que los dos primeros controles tienen un mejor desempeño, según lo expresan los autores.

En el año 2003 S. Suzuki, K. Furuta y Y. Pan [16] llevan a cabo un estudio para el levantamiento y estabilización del péndulo invertido rotacional y su propuesta es de la siguiente forma: primero se utiliza el método de estabilización no lineal global, cuyo fin es convertir un problema no lineal en un sistema lineal y obtener las soluciones a las ecuaciones de Riccati utilizadas por un regulador óptimo dependiente de estados y lo segundo es el diseño de un controlador de estructura variable por deslizamiento de sector; éste resulta ser un control que resuelve el problema de levantamiento y estabilización sin conmutación; los resultados demuestran que es una estrategia de control muy robusta y con buen desempeño ante la presencia de perturbaciones, según el artículo reportado.

En el 2007, Mojtaba Ahmadieh Khanesar, Mohammad Teshnehlab y Mahdi Aliyari Shoorehdeli [17], presentan un trabajo donde se muestra que el diseño de una estrategia de control por modos deslizantes para sistemas sub actuados inestables, de fase no mínima es complejo; lo primero que se realiza es la especificación del desempeño deseado y basado en ese desempeño se diseñan dos superficies de deslizamiento y el sistema se controla por la definición apropiada de una función de Lyapunov; ésta función hace más énfasis en el control del péndulo invertido que en el control del motor eléctrico CC.

Existe interés en la comunidad de control por el diseño de estrategias de control basado en modos deslizantes por su 
gran robustez y rechazo a los disturbios y se presenta otro trabajo de este tipo el mismo año, trabajo realizado por Masaki Izutsu, Yaodong Pan y Katsuhisa Furuta [18], aquí se propone un nuevo diseño de control llamado el estabilizador de seguimiento de modelo (Model Following Stabilizer), este método logra la estabilización de la articulación sub actuada; para el diseño del estabilizador de seguimiento de modelo se recurre al control por modos deslizantes y para el diseño de la superficie de deslizamiento se hace uso de las ecuaciones de Riccati dependientes de estado; por último el modelo de seguimiento se forma a partir de un modelo del péndulo de Furuta bajo Gravedad Artificial, se realiza simulación y experimentación.

Otro trabajo es presentado por L. Xiao, H. Su y J. Chu 2007 [19], aquí se introduce una estrategia de control predictivo en el control por modos deslizantes conocida como SMPM por sus siglas en inglés (Sliding Mode Predictive Model). Aquí se tiene en cuenta un sistema de incertidumbres acopladas no lineales en tiempo discreto, se realiza una realimentación y corrección del SMPM; según los autores el análisis en lazo cerrado demuestra que existe gran robustez en la estabilización. También en este año se realizan estudios comparativos para el levantamiento y estabilización de un péndulo de Furuta por J.A. Acosta, J. Aracil y F. Gordillo, 2007 [20], este trabajo presenta un control basado en el método denominado Gradiente de Velocidad o SG (Speed Gradient) a este control se le denomina control híbrido, se utiliza para el levantamiento y posterior estabilización del péndulo de Furuta. Para el levantamiento se concibieron controles basados en modelos de 4 y 2 dimensiones. Para la estabilización se genera un control basado en energías (energy Shaping) y un control LQR, se realiza un estudio comparativo entre las estrategias de control y se logra demostrar la efectividad y robustez del controlador basado en el modelo de 4-dimensiones.

En el año 2009, aparece en la literatura de la comunidad de control el trabajo realizado por T. C. Kuo, Y. J. Huang y B. W. Hong [21], donde se diseña un PID adaptativo por modos deslizantes y se logra convergencia y estabilidad, que es demostrada según el teorema de Lyapunov.

En el año de 2011 Ferreira Alejandra, Javier Bejarano y Fridman Leonid [22], presentan un trabajo donde se estudia el problema del control de la salida exacta robusta para un sistema lineal con entradas desconocidas, los observadores por modos deslizantes de alto orden proporcionaron una observación exacta e identificación de la entrada desconocida, se propone una metodología para seleccionar la estrategia de control más adecuada para la salida con compensación de las perturbaciones. Se realiza simulación y experimentación.

En marzo de 2012, Ashrafiuon Hashem y Whitman Alan [23], presentan un artículo donde se obtiene una solución analítica aproximada para la dinámica en lazo cerrado para la fase de deslizamiento de un péndulo invertido rotacional controlado por modos deslizantes basado en el método de escala múltiple; la expresión analítica se obtiene de la solución no lineal de la dinámica en lazo cerrado de orden reducido y esta provee los parámetros que garantizan la estabilización y mejora del desempeño del controlador.

En este mismo año también aparece el trabajo de un control por cascada de modos deslizantes óptimo, para la estabilización de un sistema sub actuado (péndulo invertido rotacional). Una estructura de control predictivo en tiempo discreto es utilizada para optimizar y obtener los parámetros y especificar las superficies que logren el objetivo de desempeño; los parámetros de la superficie están sujetos a las restricciones físicas como la saturación del sistema, se logra demostrar la estabilidad nominal y robustez del control. Este trabajo es desarrollado por Muske Kenneth R.; Ashrafiuon Hashem; Nersesov Sergey; et al. [24].

Chen Y., Huang A. en el año 2014, publican los resultados de un tipo de diseño de control adaptativo donde se consideran incertidumbres variantes en el tiempo, además de las perturbaciones. Estos autores lo primero que realizan es el desacople de la dinámica sub actuada por medio de la transformación de Olfati. Se diseña el controlador adaptativo y luego se realiza la prueba de estabilidad en lazo cerrado mediante el teorema de Lyapunov. Se realiza experimentación y simulación [25].

\section{Control basado en análisis geométricos.}

El equipo de investigadores conformado por Zhang Juan, Chen Jie y Dong Lingxun [26] en el año de 2003, reportan un artículo donde proponen un nuevo método que simplifica el análisis matemático que anteriormente se basaba en técnicas elipsoidales; esta técnica se basa en el análisis de poli topos y poliedros (objetos geométricos en tres dimensiones con caras planas).

En al año 2007, se retoma el diseño de un control basado en el análisis geométrico. Se utiliza un algoritmo de control del sistema con las restricciones basadas en la geometría del poli topo y poliedro, trabajo realizado por Zhang Juan, Chen Jie y Dong Lingxun [27]; aquí se muestra una gran robustez de la estabilización frente a disturbios.

Zhang Juan, Chen Jie y Li Peng [28], en el 2008, muestran un estudio sobre un péndulo invertido con obstáculo fijo que actúa cuando el péndulo está en su posición vertical y realiza una trayectoria planeada, la idea es la estabilización utilizando una técnica geométrica basado en poli topo y poliedro.

En el año de 2009 se propone una clase de diseño basado en la geometría del problema, gracias a las restricciones, este trabajo reportado por Pedro X. La Hera, Leonid B. Freidovich, Anton S. Shiriaev y Uwe Mettin, 2009 [29]. Aquí se proponen curvas homoclínicas pre planeadas de acuerdo a la relación entre las coordenadas generalizadas y las restricciones Holo nómicas virtuales; cuenta con un modelo de fricción en el torque, se asegura la estabilidad exponencial local. Se encuentra una discrepancia entre el experimento y el movimiento deseado, debido a que no se compensa en forma apropiada la fricción del torque. 


\section{E. Control difuso.}

El control difuso se basa en la lógica difusa que se puede describir como un sistema interpretativo donde existe una relación entre los elementos con conjuntos frontera que no están definidas en forma clara (borrosa). Estos sistemas difusos están basados en reglas descriptivas con operadores lógicos tradicionales como SI-ENTONCES, que es realmente un conocimiento heurístico y permiten generar una actuación deseada [30]. Sobre este tipo de control se han reportado una gran variedad de trabajos y se procederá a describir algunos de estos artículos.

En 1996 se reporta el trabajo de Wang H.O. et al. Donde se presenta una metodología para la estabilización de un péndulo invertido, aquí se muestra el modelo no lineal de la planta mediante el modelo de Takagi-Sugeno y se diseña el controlador difuso basado en el modelo mediante el concepto de compensación distribuida en paralelo, se realiza experimentación [31].

En el año 2006 se presenta el trabajo de Haishan Ding, Yinpeng Li, y Jianqin Mao [32]; aquí se muestra el diseño de un control proporcional para el levantamiento o swing up que va de acuerdo al signo del desplazamiento angular y la velocidad; para la estabilización se utiliza un modelo de control basado en control difuso, utilizando la técnica de compensación distribuida paralela (siglas en inglés PDC, Parallel Distributed Compensation); se logra robustez, reportan los autores.

En el año 2007 se encuentra el trabajo reportado por Yasar Beceriklia y B. Koray Celik [33], donde se muestra el control de un péndulo invertido por medio de control difuso y se aborda por medio de dos estados el diseño del control y luego la implementación usando programación en JAVA, se realiza solamente simulación.

Para el 2013 se reporta el artículo de Arpit Jain, et al [34]. Donde se propone una aproximación a un control inteligente para un péndulo invertido. Este controlador difuso es un control inteligente basado en el modelo de la lógica difusa, donde no se requiere un modelo matemático preciso o procesos computacionales complejos y puede manejar sistemas no lineales sin necesidad de linealizar. Se realiza experimentación y simulación en MATLAB SIMULINK.

\section{F. Otros tipos de estrategias de control.}

En las pasadas secciones se realizó una descripción de las estrategias de control más utilizadas por la comunidad de control y se realizó una síntesis con las tendencias o agrupación de las más comunes. En este apartado se describe brevemente otro tipos de estrategias de control que también se encuentran en la literatura y que son de gran interés.
En el 2004 se propone una estrategia para el levantamiento del péndulo basado en un PID más un controlador de impulso paso-paso, en donde se sintoniza el controlador de manera iterada hasta obtener el mínimo tiempo para el swing up, trabajo realizado por Zhongmin Wang, Yang Quan Chen y Ning Fang [35].

Otro trabajo que despierta interés se presenta este mismo año, es el de Kazuhiro Yubai, Kazunori O, y Junji Hirai, [36]; se realiza un control por Ganancia Programada del Peso (Gain-Scheduling of Weight) y un control $\mathrm{H}_{\alpha}$ con moldeo del lazo (Loop Shaping).

En el año de 2008, se presenta el trabajo propuesto por Roland Lenain, Anders Robertsson, Rolf Johansson, Anton Shiriaev y Michel Berducat [37], en este trabajo se tienen en cuenta los efectos de la fricción, fenómeno importante en el control de los sistemas robóticos. Este tema es de interés por la demanda de precisión que requiere la eliminación de los disturbios de la fricción; para esto se utilizan diferentes modelos y cada modelo tiene parámetros que deben ser encontrados (LuGre, Dahl), lo mismo que deben incluirse señales y sensores adicionales; para evitar esto se propone un observador adaptativo de dos niveles para reconstruir la velocidad y la estimación de la fricción en la articulación de los dos elementos del péndulo de Furuta por medio de un compensador basado en el modelo de fricción, es difícil la obtención de los parámetros y los investigadores solo usan el modelo de Dahl.

En este año también se realiza un estudio de un control por medio del método de linealización de la retroalimentación entrada-salida (siglas en inglés, IOFL, Input Output Feedback Linearization), usando algoritmos genéticos binarios, que es un método estocástico global y emula el proceso natural de evolución, Iraj Hassanzadeh y Saleh Mobayen [38].

En el año de 2011, Tu Yi-Wei; Ho Ming-Tzu [39], presentan un estudio sobre un péndulo invertido rotacional, donde se diseña e implementa un control visual en tiempo real con co-procesador de imagen basado en un FPGA (Field Programmable Gate Array), la posición del péndulo es medido por este medio. El péndulo utilizado en el presente estudio es más pequeño que otros, de publicaciones anteriores, lo que hizo más difícil el control del mismo, según lo expresan los autores. Los algoritmos del procesamiento de imágenes son llevadas al FPGA. Para lograr la estabilización del sistema se propuso una mezcla de un control $\mathrm{H}_{2} \mathrm{H}_{\alpha}$. La cual se resuelve usando la aproximación de la desigualdad matricial lineal (siglas en inglés LMI linear Matrix Inequality). Se realiza simulación y experimentación.

\section{G. Modelado del péndulo de Furuta.}

Como se ha descrito anteriormente el péndulo invertido rotacional simple es un tema abierto de investigación tanto desde el punto de vista del control, como también en la metodología para obtener su modelo matemático, que va 
desde al análisis por medio de la mecánica clásica, algoritmo de Denavit-Hartemberg, hasta el planteamiento de las ecuaciones de Newton y grafos de Bond. También se encuentra en la literatura aproximaciones desde el modelo de un péndulo invertido planar (carro-riel) al péndulo invertido rotacional.

En el año 2008 se presenta el estudio del modelado del péndulo de Furuta por M. Roman, E. Bobasu, y D. Sendrescu [40], en este trabajo se muestra una aproximación a su modelado a partir de los Grafos de Bond; se construyen sub modelos de cada parte del péndulo y luego se combinan para la construcción total del modelo, para lograr esto se utilizó el flujo de información y energía, se realiza únicamente simulación.

En el año 2010 apareció nuevamente interés en el modelado del péndulo de Furuta, así se realizó un estudio (reporte técnico de la universidad de Adelaida, Australia) [41] donde se obtuvo el modelo matemático del péndulo invertido rotacional por medio de dos métodos, el primero es a partir de la formulación del Lagrangiano y el otro con el método iterativo de Newton-Euler. Se realizaron aproximaciones a la dinámica completa la cual converge a la expresión comúnmente presentada para el modelo, el trabajo fue presentado por Ben S. Cazzolato y Zebb Prime.

\section{ARTICULOS DE PÉNDULOS INVERTIDOS EN COLOMBIA}

La comunidad de control en Colombia no ha sido ajena al interés de utilizar el péndulo invertido como herramienta de investigación, propósitos educacionales, punto de prueba y referencia para estrategias de control. Es así como en el año del 2002 se presenta un trabajo de tesis de pregrado en la Universidad Nacional de Colombia, donde se muestra el diseño de un control Neuro difuso aplicado a un péndulo invertido rotacional, Palacio Uribe Rodrigo Alberto [42]. Este mismo año se presenta otra tesis de pregrado por la misma universidad donde se muestra el diseño de control por medio de algoritmos genéticos para un péndulo invertido, Arenas Sánchez Andrés Fernando [43]. La Universidad EAFIT, también se interesa por este tipo de investigaciones en control y se presenta el trabajo de Javier sierra, donde se realiza un control adaptativo sobre un péndulo invertido rotacional, 2002. [44].

La universidad Autónoma de Occidente en el año 2006, incursiona en el uso del péndulo invertido como punto de investigación y presenta el trabajo de tesis consistente en el diseño e implementación de un péndulo invertido rotacional configurable, Osorio Carlos Andrés, [45].

En el año 2007 la universidad EAFIT, presenta los trabajos de tesis: Identificación Paramétrica del Modelo Matemático de un Péndulo Invertido, Estrada John Jairo [46], lo mismo que el trabajo Modelo Matemático y Control de un Péndulo Invertido, González Jorge Mario y Pineda Fabio Antonio [47].
En las revistas nacionales de Ciencia y Tecnología, también aparecen trabajos sobre el péndulo invertido, de este modo se presenta un artículo sobre el control de un péndulo por realimentación de estados utilizando algoritmos genéticos, Ibarguen Francisco, Hoyos José Gabriel [48]. En el año 2007 también se realiza otro trabajo sobre péndulos como: Control Global de un péndulo con rueda de reacción, donde se utiliza una técnica no lineal conocida como Linealización por realimentación, trabajo presentado por Buitrago Didier, Correa Víctor Daniel y Molina Alexander [49].

Otro trabajo que se encuentra es la tesis de maestría de la Universidad de los Andes llamado Modelado, Diseño y Simulación de un sistema de control para un péndulo doble, realizado por Germán Velandia Peláez 2007 [50].

Carlo Andrés Osorio, Tesis de Maestría, Universidad Nacional 2008 [51] realiza un estudio de estrategias de control lineal y no lineal considerando y sin considerar efectos de la fricción, los modelos de fricción, donde los parámetros son identificados por experimentación. Los resultados demuestran que la estrategia de control mejora considerablemente cuando se tienen en cuenta el modelo de fricción.

\section{CONCLUSIONES}

A partir de la revisión anteriormente descrita, se obtiene un documento que aporta un estado del arte, una panorámica general, que sirve como un punto de referencia para los diseñadores de control interesados en el trabajo con sistemas sub actuados como el péndulo invertido rotacional, además se evidencian las tendencias y el desarrollo de las investigaciones desde la creación del péndulo invertido rotacional hasta la actualidad y se concluye lo siguiente:

El control basado en el análisis de la energía es muy práctico y eficiente, además de que las funciones energéticas que son funciones escalares son fáciles de describir, razón por la cual esta estrategia de control es una de las más utilizadas, tanto para el levantamiento como para la estabilización del péndulo invertido rotacional.

Los controles conmutados son muy comunes, la dificultad radica en la realización del cambio de un control a otro. Para llevar a cabo esta acción, las regiones de actuación de cada control deben estar muy bien caracterizadas y determinadas.

El control sin conmutación, para lograr el objetivo de swing up y la estabilización es bastante complejo y requiere alto esfuerzo de control.

El control por estructura variable presenta gran robustez, pero se nota un gran problema de chattering.

La tendencia de las nuevas teorías de control conduce a pensar en involucrar los efectos de la fricción por la creciente necesidad del mejoramiento de la precisión con ayuda del diseño de compensadores. 
El diseño de compensadores se puede abordar de dos maneras: la primera se fundamenta en la escogencia del modelo de fricción que más se ajuste y por métodos experimentales encontrar sus parámetros. La segunda forma es identificar mediante una metodología el tipo de fricción que actúa sobre el mecanismo junto con sus parámetros y luego diseñar el compensador.

En la actualidad existen diversos tipos de péndulos que se utilizan en los laboratorios de control, entre los péndulos invertidos encontramos: el péndulo planar (carro-riel), el péndulo rotacional (dos brazos unidos mediante un pivote), este tipo de péndulo puede ser simple (un solo péndulo) o compuesto (más de un péndulo, doble, triple, etc.).

Para el modelado del péndulo de Furuta encontramos lo siguiente: Grafos de Bond, Ecuaciones de Euler-LaGrange, método de Newton iterado, Algoritmo de DenavitHartemberg, aproximación del péndulo planar al péndulo rotacional.

\section{REFERENCIAS BIBLIOGRÁFICAS.}

[1] O. Katsuhiko, Ingeniería de control moderna, vol. I. Prentice Hall, tercera edición, pp. 86. 1998.

[2] K. Furuta, M. Yamakita and S. Kobayashi, "Swing up Control of Inverted Pendulum", Journal of Systems and Control Engineering, 206(6), pp. 263-269, 1991.

[3] J. J Slotine and W. Li, Applied Nonlinear Control, pp. 193-194, Prentice Hall, 1991.

[4] K. J Åström and K Furuta, "Swinging Up a Pendulum by Energy Control", Proceeding of World Congress IFAC $13^{\text {th }}$, San Francisco California, 1996.

[5] F. Gómez Stern, "Control de Sistemas No Lineales Basado en estructura Hamiltoniana", Tesis Doctoral, Universidad de Sevilla, España, Septiembre 2002.

[6] S. Nair and N.E. Leonard, "A Normal Form for Energy Shaping: Application to the Furuta Pendulum", Proceeding of the 41st conference on decision and automation IEEE, Las Vegas, Nevada USA, December 2002.

[7] K. Machleidt, J. Kroneis and S. Liu, " Stabilization of the Furuta Pendulum Using a Nonlinear Control Law based on the Method of Controlled Lagrangians", Institute of Control Systems Technical University of Kaiserslautern, Germany, IEEE 2129-2134, 2007.

[8] L. Freidovich, A. Shiriaev, F. Gordillo, F Gómez-Estern and J. Aracil, " Partial Energy Shaping Control for Orbital Stabilization of high Frequency of Furuta Pendulum", Proceeding of the 46th conference IEE on decision and control, New Orleans, USA, December, 2007.

[9] K. J. Åström, J. Aracil and F. Gordillo, “ A family of Smooth Controllers for Swinging up a Pendulum”, Lund
University, Lund Institute of Technology, Automatic 44, 184-1848. 2008.

[10] V. Sukontanakarn and M. Parnichkun, "Real Time Optimal Control for Rotary Inverted Pendulum", Mechatronic, School of Engineering and Technology, Asian Institute of Technology, Pathumthani, Thailand. American Journal of Applied Sciences, ISSN 1546-9239, 2009.

[11] S. Awtar, N. King, T. Allen and K. Craig," Inverted pendulum systems: rotary and arm driven a mechatronic system design case study", Mechatronic 12 357-370, 2002.

[12] Y. C. Fu and J. S. Lin, "Non Linear Backstepping Control Design of the Furuta Pendulum", Proceeding of the 46th conference IEE on decision and control, Toronto, Canada, August 28-31, 2005.

[13] C. Aguilar-Ibáñez, O. F. Gutiérrez and H. SossaAzuela, "Control of the Furuta Pendulum by using a Lyapunov function", Proceeding of the 45th conference IEE on decision and control, Manchester Grand Hyatt Hotel San Diego, CA, USA, December 13-15, 2006.

[14] P. Mandic, M. Lazarevic, et al. "Real time control of rotary inverted pendulum", Annals of Engineering department, Hunedoara, International Journal of Engineering. May2014, Vol. 12 Issue 2, p211-214. 2014.

[15] J. Choi and J. S. Kim, "Robust Control for Rotational Inverted Pendulum Using Output Feedback Sliding Mode Controller and Disturbance Observer", KSME International Journal, Vol, 17 No. 10, pp. 1466-- 1474, 2003.

[16] S. Suzuki, K. Furuta and Y. Pan, "State-dependent sliding-sector VS control and application to swing- up control of Pendulum", Proceeding of the 42th conference IEE on decision and control, Maui, Hawaii USA, December 2003.

[17] M. A. Khanesar, M. Teshnehlab and M. Shoorehdeli, "Sliding Mode Control of Rotary Inverted Pendulum", University of Technology Electrical Engineering faculty Control Department, Tehran, Iran. Mediterranean Conference, July 27-29, Athens, Greek 2007.

[18] M. Izutsu and K. Furuta, "Design of a Model following stabilizer to an Artificial Gravity Control Model", SICE. Conference Annual set. 17-20, Kagawa University, Japan 2007.

[19] L. Xiao, H. Su and J. Chu, "Sliding Mode Prediction Based Control Algorithm for discrete-time Non-linear Uncertain Coupled Systems", International Journal of Control Vol. 80, No. 10, Octubre 1616-1625, 2007.

[20] J. A. Acosta, J. Aracil and F. Gordillo, "Comparative Study of Nonlinear Control Strategies for the Furuta Pendulum". Universidad de Sevilla, España, 2007. 
[21] T. C. Kuo, Y. J. Huang and B. W. Hong, "Adaptive PID with Sliding Mode Control for the Rotary Inverted Pendulum System", IEEE/ASME International Conference on Advanced Intelligent Mechatronics Suntec Convention and Exhibition Center, Singapore, July 14-17, 2009.

[22] F. Alejandra, J. Francisco, F. M. Leonid, "Robust Control with Exact Uncertainties Compensation: With or Without Chattering", IEEE TRANSACTIONS ON CONTROL SYSTEMS TECHNOLOGY Volume: Issue: 5 Pages: 969-975, 2011.

[23] H. Ashrafiuon and A. Whitman, "Closed-Loop Dynamic Analysis of a Rotary Inverted Pendulum for Control Design", JOURNAL OF DYNAMIC SYSTEMS MEASUREMENT AND CONTROL-TRANSACTIONS OF THE ASME Volume: 134 Issue: 2, March, 2012.

[24] H. Ashrafiuon, K. Muske and S. Nersesov, "Optimal Sliding Mode Cascade Control for Stabilization of Under actuated Nonlinear Systems". JOURNAL OF DYNAMIC SYSTEMS MEASUREMENT AND CONTROLTRANSACTIONS OF THE ASME Volume: 134 Issue: 2, JUN 2012.

[25] Y. F. Chen and A. C. Huang, "Adaptive control of rotary inverted pendulum system with time-varying uncertainties". SPRINGER, NONLINEAR DYNAMICS; APR, 2014, 76 1, p95-p102, 8p. 2014.

[26] Z. Juan, Ch. Jie and D. Lingxun, "Fast algorithm for constrained linear system control via geometric techniques", Control Theory and applications V01. 210 No. 5, Oct, 2008.

[27] Z. Juan, Ch. Jie and D. Lingxun, "Research on Control of Rotary Inverted Pendulum via Polytope Techniques", 2007 IEEE International Conference on Control and Automation, Guangzhou, China, May 30 to June 1, 2007.

[28] Z. Juan, Ch. Jie and L. Peng, "Obstacle Rotary Inverted Pendulum Control via Polytope Techniques". Department of Automatic Control, School of Information Science and Technology Beijing Institute of Technology Beijing, China 2008.

[29] P X. La Hera, L B. Freidovich, A. S. Shiriaev, and U. Mettin, "New Approach for Swinging up the Furuta Pendulum: Theory and experiments". Department of Applied Physics and Electronics, Umea University, Sweden 2009.

[30] Z. Yeh and K. Li, "A systematic approach for designing multistage fuzzy control systems, Fuzzy Sets and Systems, 143 (2004) 251-273, 2004.

[31] H. O. Wang and K. Tanaka, "An approach to fuzzy control of nonlinear systems: stability and design issues". IEEE Transactions on (Volume: 4, Issue: 1) Feb 1996.
[32] H. Ding, Y. Li and J. Mao, "Dynamic Switching Control for the Swing-up and Stabilizing Control of the Furuta Pendulum". University of Aeronautics and Astronautics Beijing, China 2006.

[33] Y. Beceriklia and B. K. Celik, "Fuzzy control of inverted pendulum and concept of stability using Java application". Mathematical and Computer Modeling 46 (2007) 24-37, 2007.

[34] A. Jain and D. T. Neha Sehgal, "Control of Non-Linear Inverted Pendulum using Fuzzy Logic Controller". International Journal of Computer Applications (0975 8887) Volume 69- No.27, May 2013.

[35] Z. Wang, Y. Chen and N. Fang, "Minimum Time swing up of A Rotary Pendulum by Iterative Impulsive Control". Proceeding of the 2004 American Control Conference, Boston, Massachusetts June 30 -July 2, 2004.

[36] K. Yubai, K. Okuhara and J. Hirai, "Stabilization of Rotary Inverted Pendulum by Gain-scheduling of Weight and $\mathrm{H} \infty$ Loop Shaping Controller". Mie University Department of Electrical and Electronic Engineering, Kamihama, Japan, IEEE 2006.

[37] R. Lenain, A. Robertsson, R. Johansson, A. Shiriaev and M. Berducat, "A Velocity Observer Based on Friction Adaptation". 2008 IEEE International Conference on Robotics and Automation Pasadena, CA, USA, May 19-23, 2008.

[38] I. Hassanzadeh and S. Mobayen, "GA BASED INPUT-OUTPUT FEEDBACK LINEARIZATION CONTROLLER FOR ROTARY INVERTED PENDULUM SYSTEM". Proceeding of the 5th International Symposium on Mechatronics and its Applications, Amman, Jordan, May 27-29, 2008.

[39] T. Yi-Wei and H. Ming-Tzu, "Design and implementation of robust visual servoing control of an inverted pendulum with an FPGA-based image coprocessor". MECHATRONICS Volume: 21 Issue: 7 Pages: 1170-1182, 2011.

[40] M. Roman, E. Bobasu and D. Sendrescu, "Modeling of the Rotary Inverted Pendulum System". University of Craiova, Faculty of Automation, Computers and Electronics, Department of Automation, 2007.

[41] B. S. Cazzolato and Z. Prime, "Technical Report, On the Dynamics of the Furuta Pendulum". Tech. Rep. The University of Adelaida, Australia, 24 March, 2010.

[42] R. A. Palacio Uribe, "Diseño e Implementación de un Control Neuro- Difuso aplicado a un Péndulo Invertido". Tesis de grado, Universidad Nacional, Bogotá, Colombia, 2002.

[43] A. F. Arenas Sánchez, "Generador de Estrategias de Control para un Péndulo Invertido por medio de Algoritmo 
Genético". Tesis de grado, Universidad Nacional, Bogotá, Colombia, 2002.

[44] J. Sierra, "Control adaptativo aplicado a un péndulo invertido". Tesis de grado, Maestría en Ingeniería, Universidad EAFIT, Medellín, Colombia, 2002.

[45] C. A. Osorio, "DISEÑO, CONSTRUCCIÓN Y CONTROL DE UN SISTEMA SUB-ACTUADO: PÉNDULO INVERTIDO ROTACIONAL". Tesis de grado, Ingeniería Mecatronica. Universidad Autónoma de Occidente, Cali, Colombia, 2006.

[46] J. J. Estrada, "Identificación Paramétrica del Modelo Matemático de un Péndulo Invertido". Tesis de grado Universidad EAFIT. Medellín, Colombia, 2007.

[47] J. M. González y F. A. Pineda, "Modelo Matemático y Control de un péndulo invertido". Tesis de grado Universidad EAFIT. Medellín, Colombia, 2007.

[48] F. Ibarguen y J. G. Hoyos, "Control de un Péndulo Rotacional por Realimentación de Espacios de Estado Generados a través de Algoritmos Genéticos". Scientia et Technica Año XII, No 32, Diciembre de 2006. UTP. ISSN 0122-1701, 2006.

[49] D. Buitrago, V. D Correa y A. Molina, "Control Global para un Péndulo con Rueda de Reacción". Scientia et Technica Año XIII, No 35, Agosto de 2007. Universidad Tecnológica de Pereira. ISSN 0122-1701, 2007.

[50] G. Velandia Peláez, "Modelamiento, diseño y simulación de un sistema de control para un sistema de péndulo doble invertido". Tesis de gado, Maestría en Ingeniería. Universidad de los Andes, Bogotá, Colombia, Grupo de Investigación en Automatización y Producción, 2007.

[51] Carlos Andrés Osorio, "Diseño, Construcción y Control de un Péndulo Invertido Rotacional Utilizando Técnicas Lineales y No Lineales". Tesis de grado, Maestría en Ingeniería. Universidad Nacional, Departamento de Ingeniería en Automática. Bogotá D.C., 2008. 